\title{
Retrieval of Images using Combination of Features as Color, Color Moments and Hu Moments
}

\author{
${ }^{1}$ R Rajkumar, ${ }^{2}$ Dr. M V Sudhamani \\ ${ }^{1}$ VTU - Research Scholar, Assistant Professor, Department of IS\&E, RNSIT, Bengaluru; \\ ${ }^{2}$ Professor \& Head, Department of IS\&E, RNSIT, Bengaluru; \\ raj@rnsit.ac.in; mvsudha_raj@hotmail.com;
}

\begin{abstract}
In today's digital era, several of the image retrieval systems focus on retrieving images using features from images themselves such as color, shape and textures and are referred as low-level features. In this proposed work, the features like color with HSV color space, color moments and Hu moments are employed to retrieve similar images. Various experimentations were conducted on Wang's database images to test the combination of features for higher performance using precision, recall, accuracy and f-score. The results obtained are compared with one another and also with existing works. The retrieval performance is found to be high for proposed system against existing works.
\end{abstract}

Keywords: HSV Color; Color Moments; Hu Moments; CBIR;

\section{Introduction}

Content based image retrieval (CBIR) is a technique used in computer vision applications, using which the similar images from large databases are found and returned as a resultant set based on an input query image. The word content refers to the features like color, shape, textures or any other descriptors that best describes the images. CBIR is used as a standard technique in most searching applications in online search engines. The earlier text based search engines were obtaining the images based on the keyword provided as input, but the images obtained were not appropriate or the accuracy rate was very low against the expected images. In order to overcome this issue, CBIR was introduced with feature extraction from the database images collected and use a similarity measure to compare the best matching images and return to the query system [1].

In this proposed work, we have used the standard ground truth dataset of Wang's database images that comprises of 10 classes with 100 images in each class, making a total of 1000 images in the database $[1,5]$. The feature vector for the entire database is collected, processed and stored as feature repository for future reference during querying of images.

In this proposed work, we have used only low-level features of images such as color feature, color moments and the Hu moments that describe the shape. Several experiments have been carried out and distance metric is used to compare the similarity between the database image features with query image features.

The organization of the paper is as follows: section 2 briefs about the related work, section 3 proposed a new CBIR system with an architecture and the different feature extraction techniques, the next section 4 presents the experimental results and discussions, and the final section 5 provides the conclusions and future work. 


\section{Related Works}

The image retrieval system can be implemented in various ways. The system varies with respect to types of features, number of features, combination of features, distance metrics, Graphical User Interface (GUI), indexing, relevance feedback and the database of images $[17,18]$.

A combination of features using Annular color histogram in HSV color space, color distribution entropy (CDE), the color level co-occurrence matrix (CLCM) for texture feature and the $\mathrm{Hu}$ - invariant moments as shape features are used as feature vector in [2]. A given digital color image is in RGB format as the default color space. In order to make it more convenient depending upon the applications, other color spaces can be used by conversion of RGB to either HSI, HSV, YUV etc., color spaces and are described in [3]. Out of these different color spaces, HSV color space with hue, saturation and value or intensity or brightness are more like the human eye perception of images.

The most popular color feature being used is the HSV color histogram by various researchers. The HSV color histogram feature alone is not enough to provide all the features in diversified image categories. In order to overcome such a limitation, combination of other low-level features is considered to form the feature vectors. One such combination is the texture features obtained by applying the Gabor filters from segmented regions of an image is discussed in [4]. In [5], uses a different combination of image features such as color volume with edge information, the energy feature from Gray-Level Cooccurrence Matrices (GLCM) and salient structure histogram, to retrieve the similar images.

The texture information from GLCM matrix like energy, contrast, entropy and uniformity are used as statistical information and gray color histogram is used to retrieve similar images from the dataset in [6] is discussed. The similarity is calculated with the use of distance metrics along with appropriate weights for different features. In [10], retrieval systems use color moment, local binary patterns and edge features to obtain similar images by using Manhattan similarity metric.

\section{Proposed Methods}

\subsection{Architecture of the system}

To implement any CBIR system, there are of two phases namely, online and offline phases. In the proposed work, we have used the features as HSV color histogram, color moments and the Hu's invariant moments. The proposed architecture of the CBIR system is shown in figure 1 . In the offline phase, all the images in the repository are processed with feature extraction algorithm and the extracted features are stored for future reference. The online phase is initiated whenever the user submits a query image for which the similar images needs to be retrieved, is processed with the same feature extraction algorithms at runtime. The features obtained for the query image is compared with the features stored in the repository. The similarity metric used is Euclidean distance to get very close proximity images and then rank the images in ascending order of similarity and display the results on the GUI. 


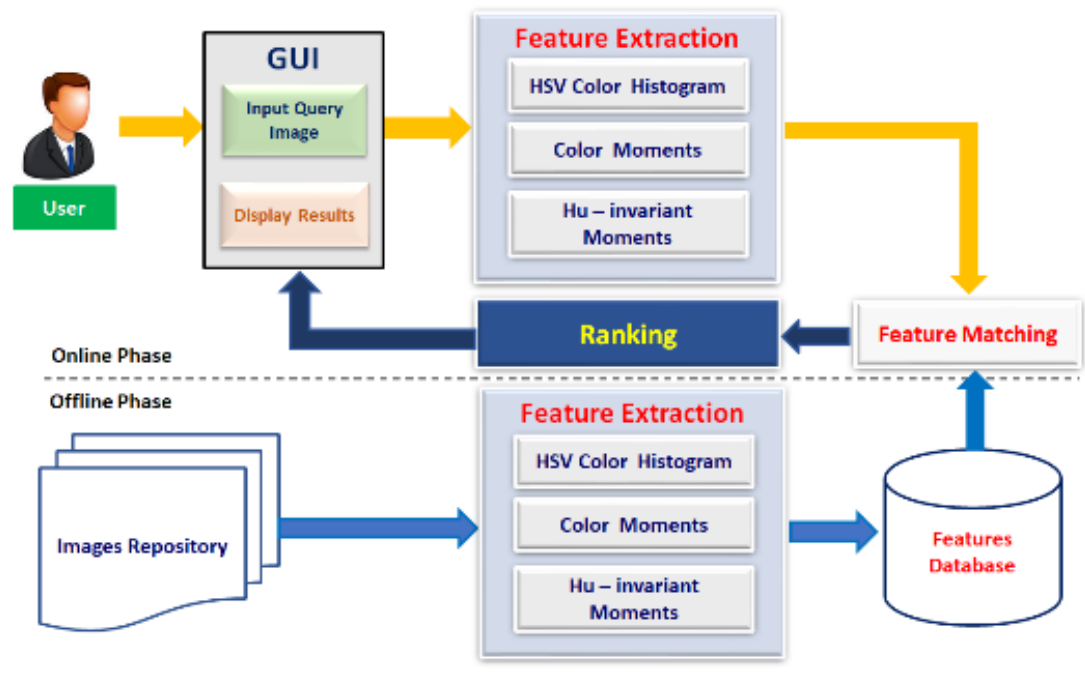

Figure 1: Proposed CBIR Architecture

\subsection{Features Extraction techniques}

\subsubsection{Color}

In digital image processing, there are different color space models available. The RGB color space is the most frequently used in generating of images from electronic devices like digital camera or a scanner. But the RGB color model is not suitable for processing of images and make analysis of it. Hence, there are several other models like HSV, HSI, CMYK, L*a*b, etc. that perceives the images in the way human eye perceives images in a natural way. In this work, we have considered the HSV color space model for feature extractions and finding out the moments in an image. The figure 2 shows HSV color model and its hexacone coordinate system $[12,13]$.
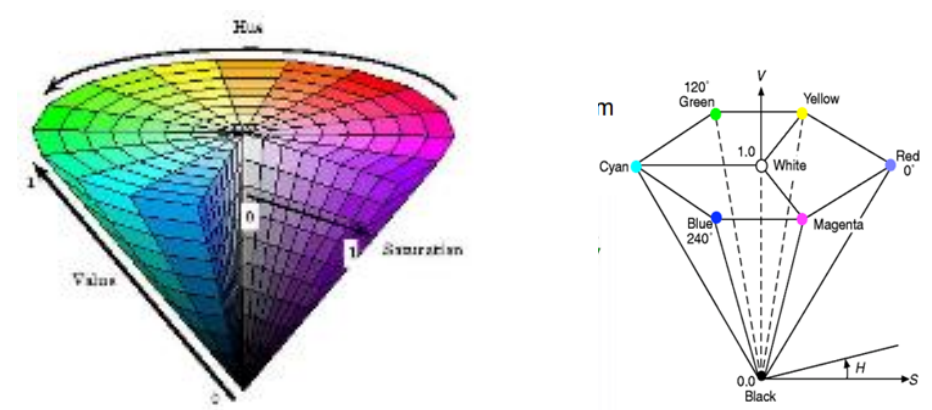

Figure 2: HSV color space - Hue, Saturation and Value [12] and hexacone coordinate system [13]

Hue is the color expressed from $0^{\circ}$ to $360^{\circ}$ degrees, the colors have the ranges as: for Red color between $0^{\circ}$ to $60^{\circ}$, Yellow color is between $61^{\circ}$ to $120^{\circ}$, Green color is between $121^{\circ}-180^{\circ}$, Cyan is between $181^{\circ}-$ $240^{\circ}$, Blue color is between $240^{\circ}-300^{\circ}$ and Magenta color is between $301^{\circ}-360^{\circ}$. Saturation is the gray color that ranges from $0-100$ in percentage, 0 being darker and 1 being brighter. The value or brightness is similar to saturation that gives the intensity or brightness of the color ranging from 0 to 100 percent, with 0 being black and 100 is brightest revealing the most color.

The HSV color feature for an image can be obtained by translating the image from RGB color space to HSV color space and is shown in equations (1) to (5). The conversion algorithm is given: 
Step 1: Normalize the RGB values from $0 . .255$ to $0 . .1$ :

$$
R^{\prime}=R / 255, \quad G^{\prime}=G / 255^{\prime}=B / 255
$$

Step 2: Compute $C \max =\max \left(R^{\prime}, G^{\prime}, B^{\prime}\right)$ and $C \min =\min \left(R^{\prime}, G^{\prime}, B^{\prime}\right)$

$$
\Delta=C \max -C \min
$$

Step 3: Hue $=\left\{\begin{array}{l}60^{0} \times\left(\frac{G^{\prime}-B^{\prime}}{\Delta} \bmod 6\right), C \max =R^{\prime} \\ 60^{0} \times\left(\frac{B^{\prime}-R^{\prime}}{\Delta}+2\right), C \max =G^{\prime} \\ 60^{0} \times\left(\frac{R^{\prime}-G^{\prime}}{\Delta}+4\right), C \max =B^{\prime}\end{array}\right.$

Step 4: Saturation: $S=\left\{\begin{array}{c}0, C \max =0 \\ \frac{\Delta}{C \max }, C \max \neq 0\end{array}\right.$

Step 5: Value: $\mathrm{V}=\mathrm{Cmax}$

\section{Algorithm 1: The HSV color histogram computation as feature now computed as follows:}

Step 1: Split the images into 3 different planes as Hue H, Saturation S and Value V

Step 2: Each plane of $\mathrm{H}, \mathrm{S}$ and $\mathrm{V}$ is quantized by specifying quantization levels as of 8,4 and 2 respectively

Step 3: Compute the maximum value for each plane of $\mathrm{H}, \mathrm{S}$ and $\mathrm{V}$

Step 4: Find the indexes of all the pixel values for the quantized channel values

Step 5: Indicate the pixel value to be 1 for index with channel value not zero

Step 6: Normalize the feature vector of 64 bins to be unit sum

Thus, the above steps are used to compute the HSV color histogram of size $8 \times 4 \times 2$ equal to 64 bins of feature.

\subsubsection{Color moments}

The color moments are features of an image that are invariant towards scaling and rotation. It characterizes the color distribution of an image. The following are the color moments computed for color images:

1. Mean - it is the $1^{\text {st }}$ color moment computed as average color of the image and is given by equation (6):

$$
\mu_{i}=\sum_{j=1}^{N} \frac{1}{N} p_{i j}
$$

where $\mathrm{N}$ is number of pixels in the image and $p_{i j}$ is the value of the $\mathrm{j}^{\text {th }}$ pixel of the image at the $\mathrm{i}^{\text {th }}$ color channel.

2. Standard Deviation $-2^{\text {nd }}$ color moment and is given by equation (7):

$$
\sigma=\sqrt{\left(\frac{1}{N} \sum_{j=1}^{N}\left(p_{i j}-\mu_{i}\right)^{2}\right)}
$$

where $\mu_{i}$ is the mean value for $\mathrm{i}^{\text {th }}$ color channel. 
3. Skewness $-3^{\text {rd }}$ color moment that describes how asymmetric is the color distribution and given by equation (8):

$$
s_{i}=\sqrt[3]{\left(\frac{1}{N} \sum_{j=1}^{N}\left(p_{i j}-\mu_{i}\right)^{3}\right)}
$$

4. Kurtosis $-4^{\text {th }}$ color moment that provides the description regarding shape of the color distribution [13] and is given by equation (9):

$$
K=\frac{E(x-\mu)^{4}}{\sigma^{4}}
$$

where $\mu$ is mean of $x, \sigma$ is second color moment of $x$ and $E(t)$ expected value of quantity t.

The color moments are computed for three channels of the image in HSV color space and a total of 12 moments ( 3 channels $\times 4$ moments) are extracted as second feature vector.

\subsubsection{Hu-Invariant moments}

The $\mathrm{Hu}$ - Invariant moments of an image are invariant to transformations like translation, scaling, rotation and reflection. These moment features can be extracted using central geometric moments of an image, given by equation (10):

$$
\mu_{p q}=\int_{-\infty}^{\infty} \int_{-\infty}^{\infty}\left(x-x_{c}\right)^{p}\left(y-y_{c}\right)^{q} f(x, y) d x d y
$$

where $x_{c}=m_{10} / m_{00}, y_{c}=m_{01} / m_{00}$ are the coordinates of centroid.

The central moments in terms of geometric moments is given by equation (11):

$$
\mu_{p q}=\sum_{k=0}^{p} \sum_{j=0}^{q}\left(\begin{array}{l}
p \\
k
\end{array}\right)\left(\begin{array}{l}
q \\
j
\end{array}\right)\left(-1^{k+j}\right) x_{c}^{k} y_{c}^{j} m_{p-k, q-j}
$$

There are seven such $\mathrm{Hu}-$ moments [14] as given from equations (12) to (18):

$$
\begin{aligned}
\emptyset_{1}= & \eta_{20}+\eta_{02} \\
\emptyset_{2}= & \left(\eta_{20}-\eta_{02}\right)^{2}+4 \eta_{11}^{2} \\
\emptyset_{3}= & \left(\eta_{30}-3 \eta_{12}\right)^{2}+\left(3 \eta_{21}-\mu_{03}\right)^{2} \\
\emptyset_{4}= & \left(\eta_{30}+\eta_{12}\right)^{2}+\left(\eta_{21}+\mu_{03}\right)^{2} \\
\emptyset_{5}= & \left(\eta_{30}-3 \eta_{12}\right)\left(\eta_{30}+\eta_{12}\right)\left[\left(\eta_{30}+\eta_{12}\right)^{2}-3\left(\eta_{21}+\eta_{03}\right)^{2}\right]+\left(3 \eta_{21}-\eta_{03}\right)\left(\eta_{21}+\eta_{03}\right)\left[3 \left(\eta_{30}+\right.\right. \\
& \left.\left.\eta_{12}\right)^{2}-\left(\eta_{21}+\eta_{03}\right)^{2}\right] \\
\emptyset_{6}= & \left(\eta_{20}-\eta_{02}\right)\left[\left(\eta_{30}+\eta_{12}\right)^{2}-\left(\eta_{21}+\eta_{03}\right)^{2}\right]+4 \eta_{11}\left(\eta_{30}+\eta_{12}\right)\left(\eta_{21}+\eta_{03}\right) \\
\emptyset_{7}= & \left(3 \eta_{21}-\eta_{03}\right)\left(\eta_{30}+\eta_{12}\right)\left[\left(\eta_{30}+\eta_{12}\right)^{2}-3\left(\eta_{21}+\eta_{03}\right)^{2}\right]-\left(\eta_{30}-3 \eta_{12}\right)\left(\eta_{21}+\right. \\
& \left.\eta_{03}\right)\left[3\left(\eta_{30}+\eta_{12}\right)^{2}-\left(\eta_{21}+\eta_{03}\right)^{2}\right]
\end{aligned}
$$

\subsubsection{Similarity Measure}

The similarity metrics used in our proposed work is the Euclidean distance. There are three different features considered for experiments with individual color histogram as experiment 1 , color histogram is combined with color moments as experiment 2 and then combination of color histogram with shape features extracted with Hu moments as experiment 3 . The distances are found for individual features between query image and images in the database with weights as given in the equation (19).

$$
D_{(q, d b)}=w_{1} S_{C H i s t}+w_{2} S_{C m o m}+w_{3} S_{H u}
$$

Where,

q refers to query image 
$\mathbf{d b}$ refers to database images features respectively

The weights in experiment 1 is $w_{1}=1.00$ because of only one feature, in experiment 2 the weights are $w_{1}=0.68$ of color histogram and $w_{2}=0.32$ for color moments, in experiment 3 the weights are $w_{1}=0.73$ for color histogram and $w_{2}=0.27$ for Hu-invariant moments.

\subsubsection{Performance Evaluation Metrics}

To evaluate the performance of the CBIR system, random inputs of images as queries are submitted and based on the retrieved results, precision and recall values are calculated using the equation (20) and (21). Using the values from equations (20) and (21), accuracy and F-score are calculated using the equations (22) and (23).

$$
\begin{aligned}
& \text { Precision }=\frac{\text { Number of Relevant Images Retrieved }}{\text { Total Number of Images Retrieved }} \\
& \text { Recall }=\frac{\text { Number of Relevant Images Retrieved }}{\text { Total Number of Images in database }} \\
& \text { Accuracy }=\frac{\text { Precision }+ \text { Recall }}{2} \\
& F-\text { score }=2 \times \frac{\text { Precision } \times \text { Recall }}{\text { Precision }+ \text { Recall }}
\end{aligned}
$$

\section{$4 \quad$ Results and discussions}

\subsection{Experiment-1}

This experiment was carried out with the HSV color histogram as feature consists of 64 bins. Images from various categories are chosen randomly and are given as input to the CBIR system. The average value of precision and recall of individual category are calculated and averaged over all categories to know the overall performance of the system. The results are shown in Table 1 and the graph plot is shown in figure 3.

Table 1. Average values precision and recall with various categories for experiment-1

\begin{tabular}{|l|c|c|c|c|}
\hline \multirow{2}{*}{ Classes } & \multicolumn{4}{|c|}{$\begin{array}{c}\text { Experiment-1 } \\
\text { HSV Color Histogram }\end{array}$} \\
\cline { 2 - 5 } & Precision (\%) & Recall (\%) & Accuracy (\%) & F-Score (\%) \\
\hline Africans & 81.68 & 69.76 & 75.72 & 75.25 \\
\hline Beaches & 20.47 & 19.51 & 19.99 & 19.98 \\
\hline Buildings & 61.78 & 52.09 & 56.94 & 56.52 \\
\hline Buses & 61.20 & 58.42 & 59.81 & 59.78 \\
\hline Dinosaurs & 96.07 & 63.71 & 79.89 & 76.61 \\
\hline Elephants & 34.34 & 31.91 & 33.13 & 33.08 \\
\hline Flowers & 57.52 & 52.59 & 55.06 & 54.94 \\
\hline Horses & 90.44 & 67.42 & 78.93 & 77.25 \\
\hline Mountains & 30.61 & 29.37 & 29.99 & 29.98 \\
\hline Food & 80.20 & 74.87 & 77.54 & 77.44 \\
\hline Average & $\mathbf{6 1 . 4 3}$ & $\mathbf{5 1 . 9 6}$ & $\mathbf{5 6 . 7 0}$ & $\mathbf{5 6 . 3 0}$ \\
\hline
\end{tabular}




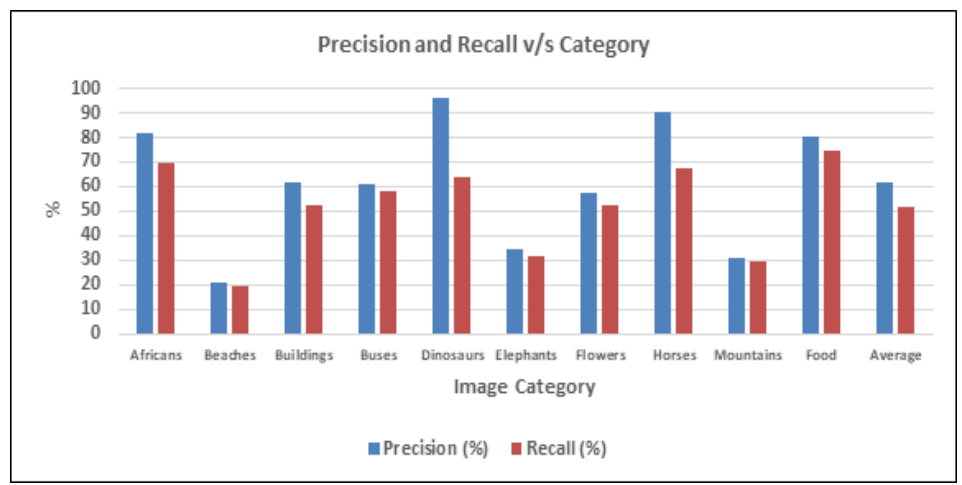

Figure 3: Performance of experiment 1

\subsection{Experiment-2}

This experiment was carried out with the HSV color histogram with 64 bins and the color moments with 12 features. The average value of precision and recall of individual category are calculated and averaged over all categories to know the overall performance of the system. The results are shown in Table 2 and the graph plot is shown in figure 4 .

Table 2. Average values precision and recall with various categories for experiment-2

\begin{tabular}{|l|c|c|c|c|}
\hline \multirow{2}{*}{ Classes } & \multicolumn{5}{|c|}{ Experiment-2 } \\
\cline { 2 - 5 } & HSV Color Histogram + Color Moments (Mean + SD + Skewness + Kurtosis) \\
\hline Africans & 53.37 & 47.07 & 50.22 & 50.02 \\
\hline Beaches & 49.31 & 47.34 & 48.33 & 48.30 \\
\hline Buildings & 38.21 & 36.23 & 37.22 & 37.19 \\
\hline Buses & 77.82 & 66.21 & 72.02 & 71.55 \\
\hline Dinosaurs & 99.76 & 69.40 & 84.58 & 81.86 \\
\hline Elephants & 70.69 & 60.60 & 65.65 & 65.26 \\
\hline Flowers & 56.24 & 53.41 & 54.83 & 54.79 \\
\hline Horses & 86.68 & 71.95 & 79.32 & 78.63 \\
\hline Mountains & 61.53 & 56.78 & 59.16 & 59.06 \\
\hline Food & 48.27 & 44.54 & 46.41 & 46.33 \\
\hline Average & $\mathbf{6 4 . 1 9}$ & $\mathbf{5 5 . 8 5}$ & $\mathbf{6 0 . 0 2}$ & $\mathbf{5 9 . 7 3}$ \\
\hline
\end{tabular}

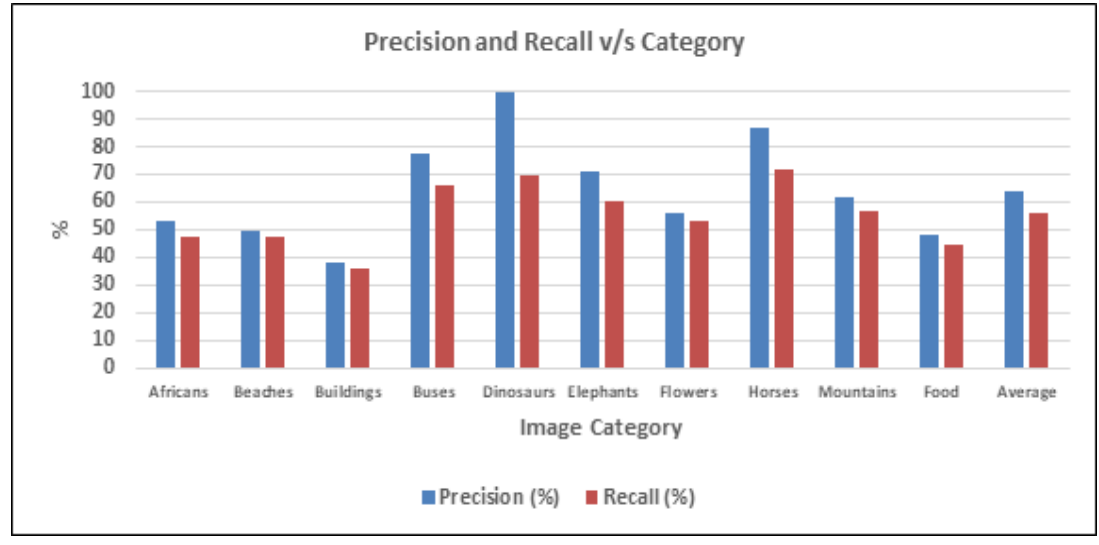

Figure 4: Performance of experiment 2 


\subsection{Experiment-3}

The experiment-3 is carried out with the HSV color histogram 64 bins and Hu-invariant moments with 7 features. The average value of precision and recall of individual category are calculated and averaged over all categories to know the overall performance of the system. The results are shown in Table 3 and the graph plot is shown in figure 5 .

Table 3. Average values precision and recall with various categories for experiment-3

\begin{tabular}{|l|c|c|c|c|}
\hline \multirow{2}{*}{ Classes } & \multicolumn{4}{|c|}{ Experiment-3 } \\
\cline { 2 - 5 } & HSV Color Histogram + Hu-Invariant Moments \\
\cline { 2 - 5 } & Precision (\%) & Recall (\%) & Accuracy (\%) & F-Score (\%) \\
\hline Africans & 88.66 & 81.63 & 85.15 & 85.00 \\
\hline Beaches & 48.00 & 48.00 & 48.00 & 48.00 \\
\hline Buildings & 77.06 & 65.03 & 71.05 & 70.54 \\
\hline Buses & 85.75 & 84.87 & 85.31 & 85.31 \\
\hline Dinosaurs & 96.59 & 69.91 & 83.25 & 81.11 \\
\hline Elephants & 53.44 & 51.82 & 52.63 & 52.62 \\
\hline Flowers & 64.82 & 63.49 & 64.16 & 64.15 \\
\hline Horses & 95.65 & 78.39 & 87.02 & 86.16 \\
\hline Mountains & 58.20 & 58.20 & 58.20 & 58.20 \\
\hline Food & 88.28 & 84.70 & 86.49 & 86.45 \\
\hline Average & $\mathbf{7 5 . 6 4}$ & $\mathbf{6 8 . 6 0}$ & $\mathbf{7 2 . 1 2}$ & $\mathbf{7 1 . 9 5}$ \\
\hline
\end{tabular}

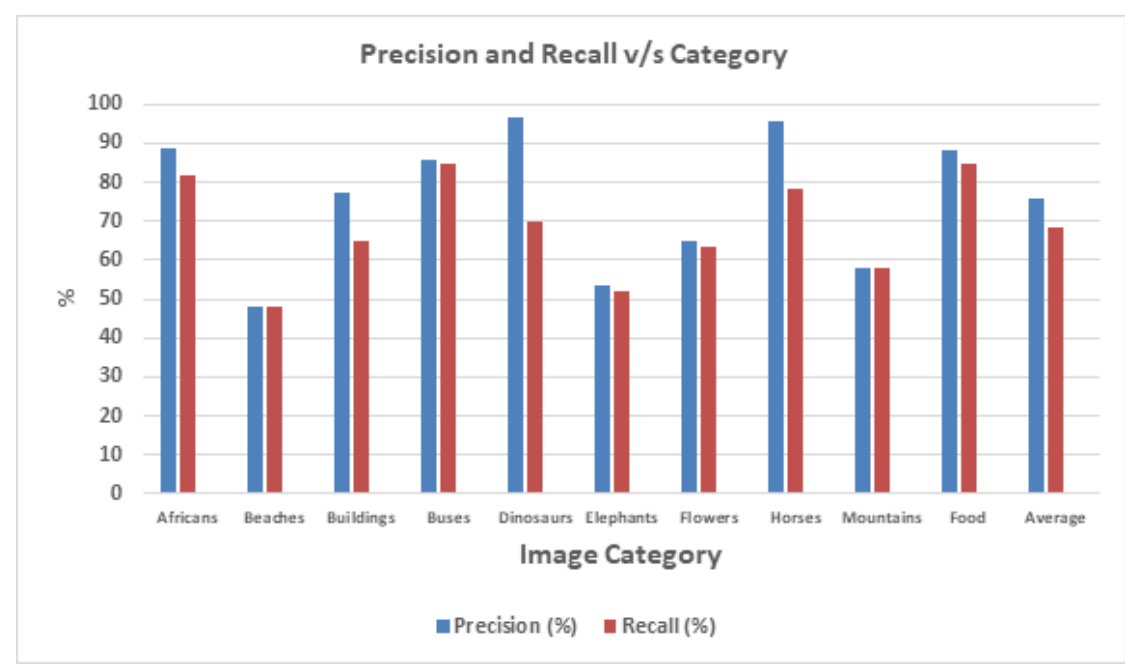

Figure 5: Performance of experiment-3

\subsection{Relative study}

\subsubsection{Self-relative study}

The performance of the experiments conducted is compared as in table 4. 
Table 4. Comparison of performance with experiments conducted

\begin{tabular}{|l|c|c|c|c|c|c|}
\hline \multirow{2}{*}{ Classes } & \multicolumn{2}{|c|}{ Experiment-1 } & \multicolumn{2}{c|}{ Experiment-2 } & \multicolumn{2}{c|}{ Experiment-3 } \\
\cline { 2 - 7 } & Precision (\%) & Recall (\%) & Precision (\%) & Recall (\%) & Precision (\%) & Recall (\%) \\
\hline Africans & 81.68 & 69.76 & 53.37 & 47.07 & 88.66 & 81.63 \\
\hline Beaches & 20.47 & 19.51 & 49.31 & 47.34 & 48.00 & 48.00 \\
\hline Buildings & 61.78 & 52.09 & 38.21 & 36.23 & 77.06 & 65.03 \\
\hline Buses & 61.20 & 58.42 & 77.82 & 66.21 & 85.75 & 84.87 \\
\hline Dinosaurs & 96.07 & 63.71 & 99.76 & 69.40 & 96.59 & 69.91 \\
\hline Elephants & 34.34 & 31.91 & 70.69 & 60.60 & 53.44 & 51.82 \\
\hline Flowers & 57.52 & 52.59 & 56.24 & 53.41 & 64.82 & 63.49 \\
\hline Horses & 90.44 & 67.42 & 86.68 & 71.95 & 95.65 & 78.39 \\
\hline Mountains & 30.61 & 29.37 & 61.53 & 56.78 & 58.20 & 58.20 \\
\hline Food & 80.20 & 74.87 & 48.27 & 44.54 & 88.28 & 84.70 \\
\hline Average & $\mathbf{6 1 . 4 3}$ & $\mathbf{5 1 . 9 6}$ & $\mathbf{6 4 . 1 9}$ & $\mathbf{5 5 . 8 5}$ & $\mathbf{7 5 . 6 4}$ & $\mathbf{6 8 . 6 0}$ \\
\hline
\end{tabular}

From the above table 4, it is observed that the experiment-1 with only color histogram feature, the performance is moderate and in experiment-2 by the combination of color histogram with color moments, there is an improvement in precision and recall by $2.67 \%$ and $3.89 \%$ respectively. The experiment-3 gives better improvement in precision and recall than experiment- 2 by $11.45 \%$ and $12.75 \%$ respectively.

\subsubsection{Relative study against existing systems}

The performance metrics of the proposed work is compared against works in [15], [16] and [6] and are shown in table 5. In the table 5, Pr represents Precision and Re for Recall.

Table 5. Comparison of performance with existing systems

\begin{tabular}{|l|c|c|c|c|c|c|c|c|}
\hline \multirow{2}{*}{ Classes } & \multicolumn{2}{|c|}{ Existing Work [15] } & \multicolumn{2}{c|}{ Existing Work [16] } & \multicolumn{2}{c|}{ Existing Work [6] } & \multicolumn{2}{c|}{$\begin{array}{c}\text { Proposed Work } \\
\text { Experiment-3 }\end{array}$} \\
\cline { 2 - 9 } & $\operatorname{Pr}(\%)$ & $\operatorname{Re}(\%)$ & $\operatorname{Pr}(\%)$ & $\operatorname{Re}(\%)$ & $\operatorname{Pr}(\%)$ & $\operatorname{Re}(\%)$ & $\operatorname{Pr}(\%)$ & $\operatorname{Re~(\% )~}$ \\
\hline Africans & 73.00 & 14.60 & 68.30 & 14.10 & 73.33 & 16.00 & 88.66 & 81.63 \\
\hline Beaches & 39.25 & 7.85 & 54.00 & 19.20 & 55.66 & 21.15 & 48.00 & 48.00 \\
\hline Buildings & 46.25 & 9.25 & 56.20 & 17.40 & 63.25 & 14.23 & 77.06 & 65.03 \\
\hline Buses & 82.50 & 16.50 & 88.80 & 12.10 & 86.75 & 21.15 & 85.75 & 84.87 \\
\hline Dinosaurs & 98.00 & 19.60 & 99.20 & 10.10 & 92.52 & 50.12 & 96.59 & 69.91 \\
\hline Elephants & 59.25 & 11.85 & 65.80 & 14.90 & 67.00 & 25.25 & 53.44 & 51.82 \\
\hline Flowers & 86.00 & 17.20 & 89.10 & 11.20 & 86.00 & 22.34 & 64.82 & 63.49 \\
\hline Horses & 89.75 & 17.95 & 80.30 & 13.40 & 75.00 & 21.66 & 95.65 & 78.39 \\
\hline Mountains & 41.75 & 8.35 & 52.20 & 21.30 & 71.23 & 15.00 & 58.20 & 58.20 \\
\hline Food & 53.45 & 10.69 & 73.30 & 13.20 & 69.25 & 21.35 & 88.28 & 84.70 \\
\hline Average & $\mathbf{6 6 . 9 2}$ & $\mathbf{1 3 . 3 8}$ & $\mathbf{7 2 . 7 0}$ & $\mathbf{1 4 . 6 0}$ & $\mathbf{7 3 . 9 9}$ & $\mathbf{2 2 . 8 2}$ & $\mathbf{7 5 . 6 4}$ & $\mathbf{6 8 . 6 0}$ \\
\hline
\end{tabular}

We can observe from the above table that, the proposed work has higher performance than existing works in [15], [16] and [6], with respect to average precision by $1.65 \%$ and average recall by $45.78 \%$ respectively. 
R Rajkumar, Dr. M V Sudhamani, Retrieval of Images using Combination of Features as Color, Color Moments and Hu Moments, Advances in I mage and Video Processing, Volume 7 No 5, October (2019); pp: $9-21$

\subsubsection{Accuracy and F-Score metrics computation}

The metrics Accuracy and F-score are computed and used here to compare existing and proposed works as shown in table 6 . The proposed work has given higher values with respect to accuracy and F-score as $23.71 \%$ and $37.07 \%$ respectively. In the table 6 , Acc represents Accuracy

Table 6. Comparison of Accuracy \& F-Score with existing and proposed works

\begin{tabular}{|l|c|c|c|c|c|c|c|c|}
\hline \multirow{2}{*}{ Classes } & \multicolumn{2}{|c|}{ Existing Work [15] } & \multicolumn{2}{|c|}{ Existing Work [16] } & \multicolumn{2}{c|}{ Existing Work [6] } & \multicolumn{2}{c|}{ Proposed Work } \\
\cline { 2 - 9 } & Acc (\%) & F-Score (\%) & Acc (\%) & F-Score (\%) & Acc (\%) & F-Score (\%) & Acc (\%) & F-Score (\%) \\
\hline Africans & 43.80 & 24.33 & 41.20 & 23.37 & 44.67 & 26.27 & 85.15 & 85.00 \\
\hline Beaches & 23.55 & 13.08 & 36.60 & 28.33 & 38.41 & 30.65 & 48.00 & 48.00 \\
\hline Buildings & 27.75 & 15.42 & 36.80 & 26.57 & 38.74 & 23.23 & 71.05 & 70.54 \\
\hline Buses & 49.50 & 27.50 & 50.45 & 21.30 & 53.95 & 34.01 & 85.31 & 85.31 \\
\hline Dinosaurs & 58.80 & 32.67 & 54.65 & 18.33 & 71.32 & 65.02 & 83.25 & 81.11 \\
\hline Elephants & 35.55 & 19.75 & 40.35 & 24.30 & 46.13 & 36.68 & 52.63 & 52.62 \\
\hline Flowers & 51.60 & 28.67 & 50.15 & 19.90 & 54.17 & 35.47 & 64.16 & 64.15 \\
\hline Horses & 53.85 & 29.92 & 46.85 & 22.97 & 48.33 & 33.61 & 87.02 & 86.16 \\
\hline Mountains & 25.05 & 13.92 & 36.75 & 30.25 & 43.12 & 24.78 & 58.20 & 58.20 \\
\hline Food & 32.07 & 17.82 & 43.25 & 22.37 & 45.30 & 32.64 & 86.49 & 86.45 \\
\hline Average & $\mathbf{4 0 . 1 5}$ & $\mathbf{2 2 . 3 1}$ & $\mathbf{4 3 . 6 5}$ & $\mathbf{2 4 . 3 2}$ & $\mathbf{4 8 . 4 1}$ & $\mathbf{3 4 . 8 8}$ & $\mathbf{7 2 . 1 2}$ & $\mathbf{7 1 . 9 5}$ \\
\hline
\end{tabular}

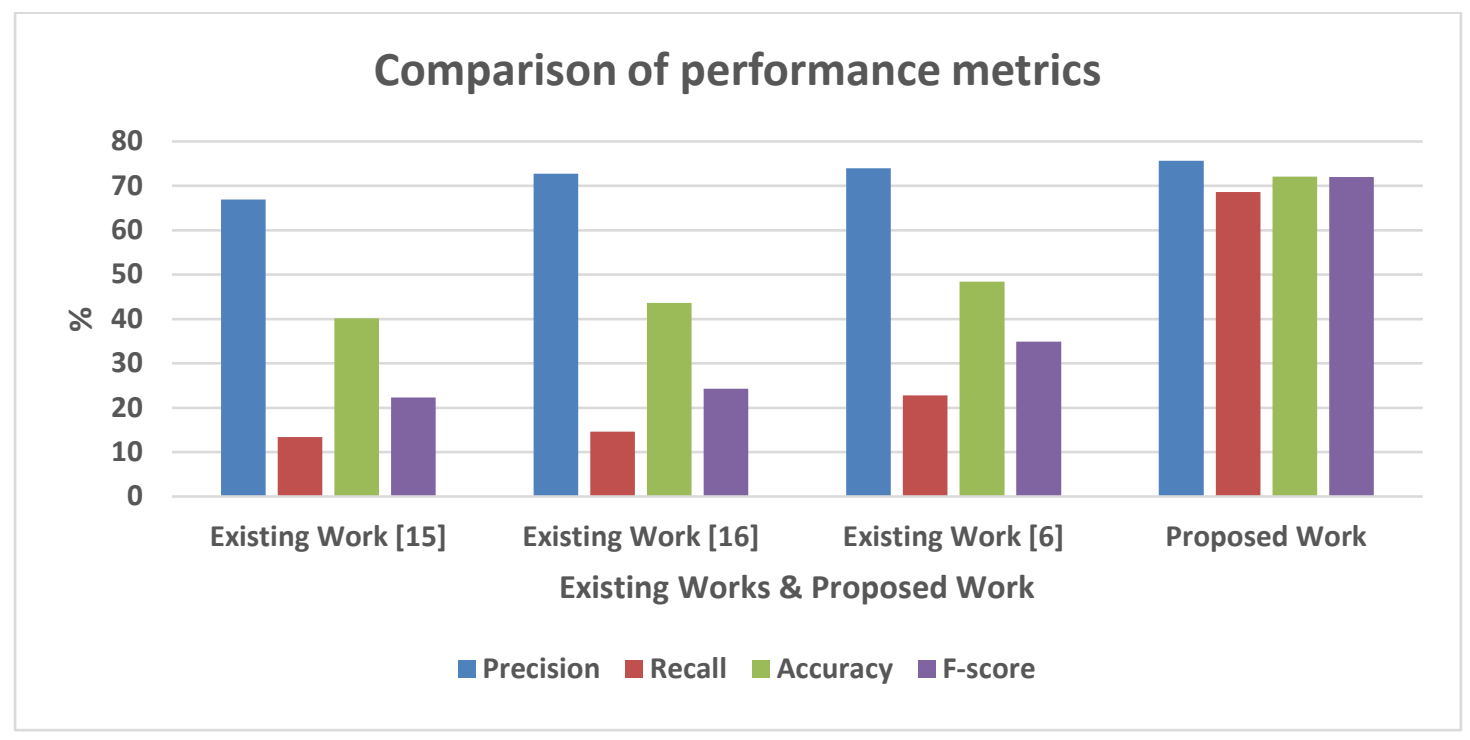

Figure 6: Comparison of performance with existing systems and proposed system

\subsection{Results}

The following figures from 7 to 18 shows sample results from the proposed work. 


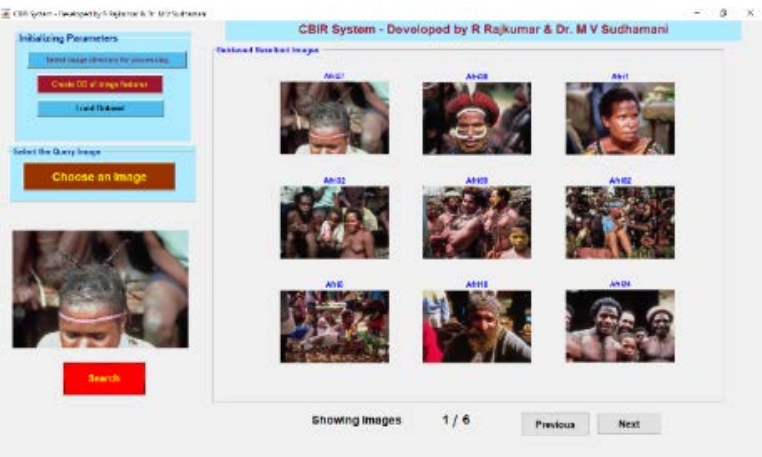

Figure 7: Query in class Africans \# 1 (pp 1)

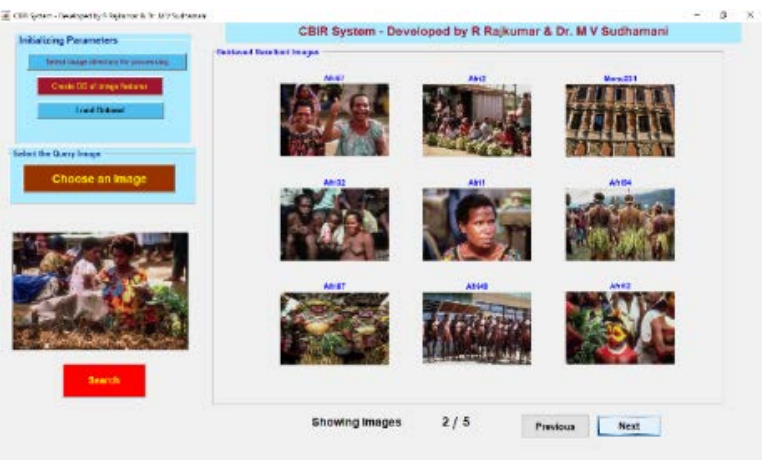

Figure 9: Query in class Africans \# 2 (pp 2)

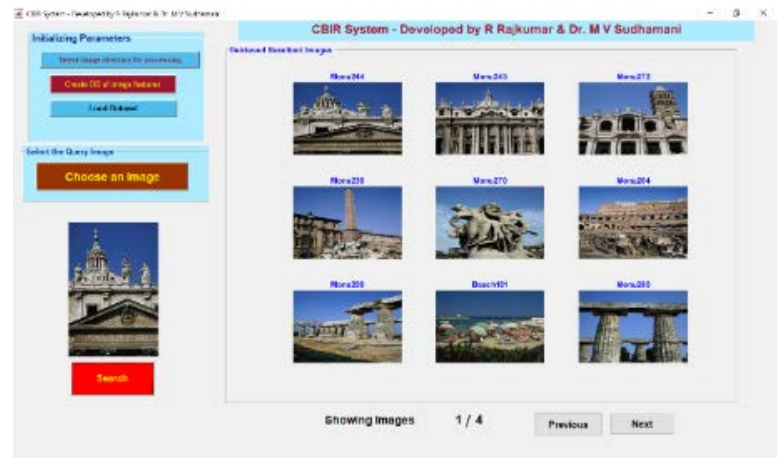

Figure 11: Query in class Buildings \# 1 (pp 1)

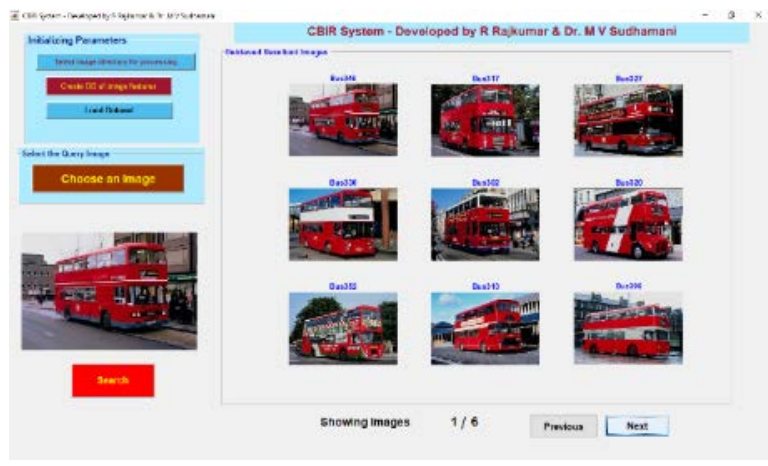

Figure 13: Query in class Bus \# 1 (pp 1)

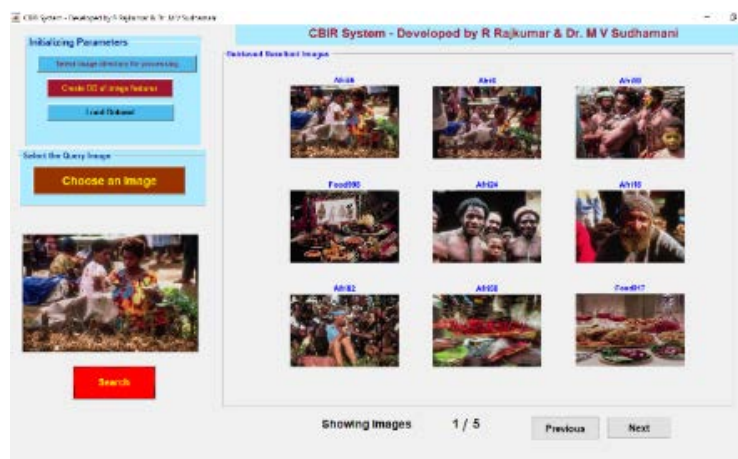

Figure 8: Query in class Africans \# 2 (pp 1)

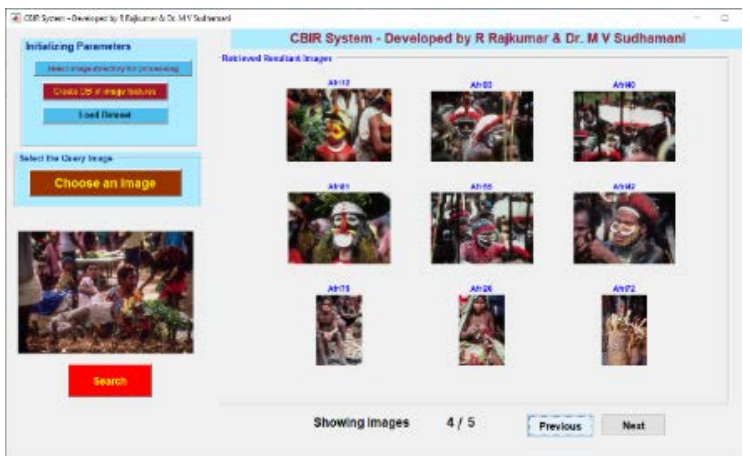

Figure 10: Query in class Africans \# 2 (pp 4)

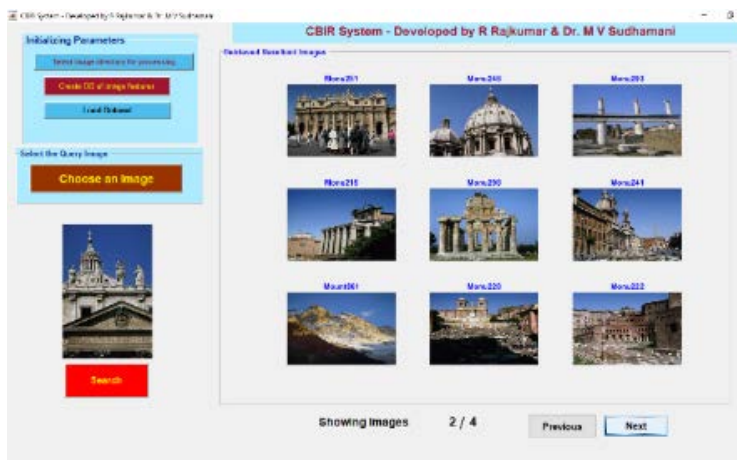

Figure 12: Query in class Buildings \# 1 (pp 2)

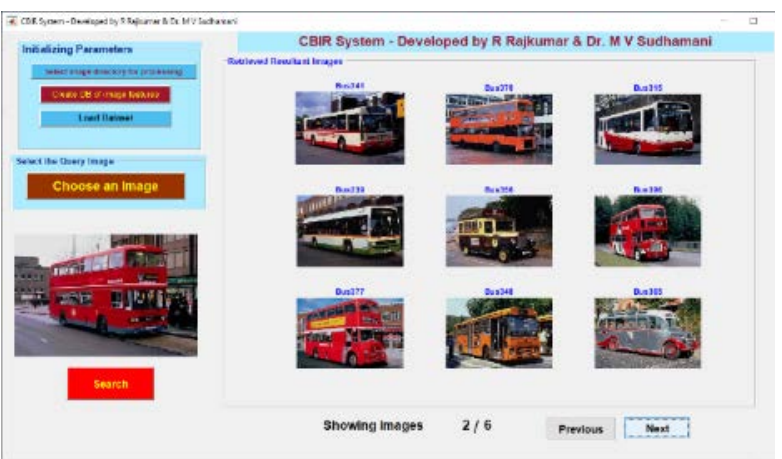

Figure 14: Query in class Bus \# 1 (pp 2) 


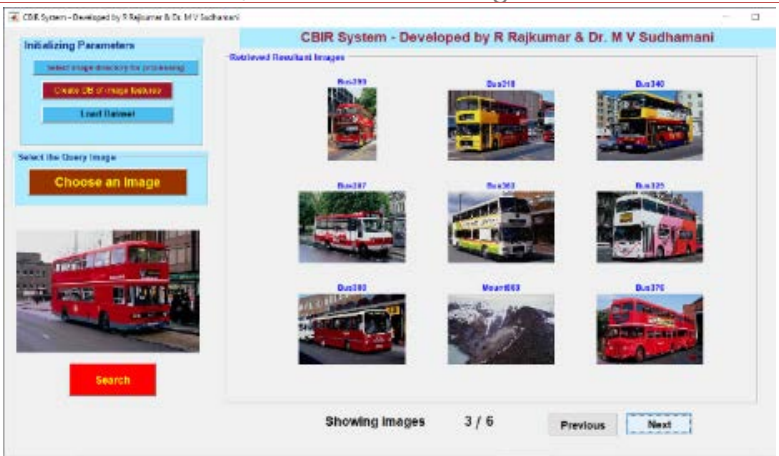

Figure 15: Query in class Bus \# 1 (pp 3)

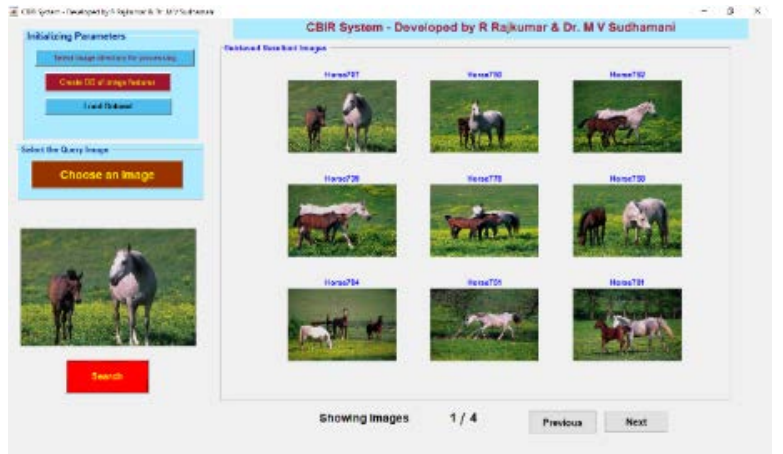

Figure 17: Query in class Horses \# 2 (pp 1)

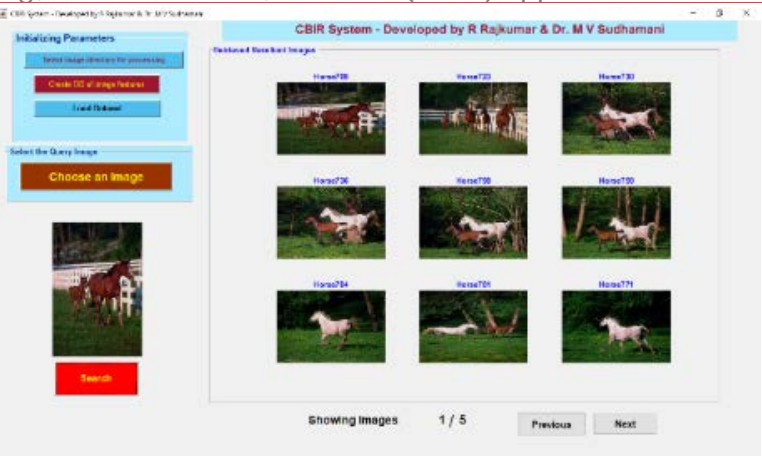

Figure 16: Query in class Horses \# 1 (pp 1)

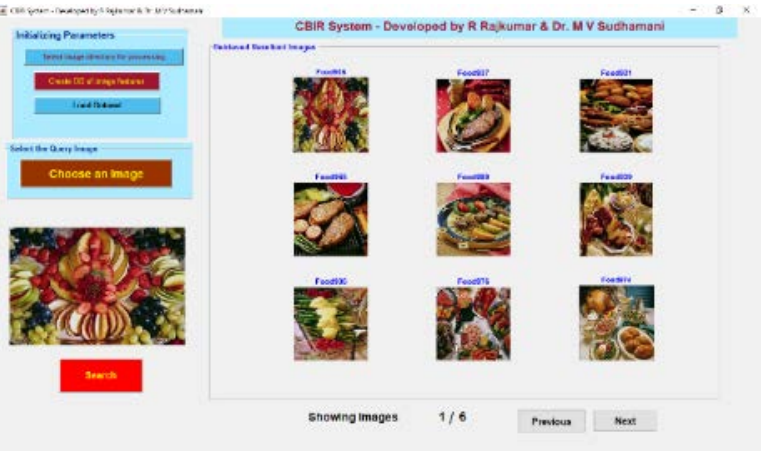

Figure 18: Query in class Food \# 1 (pp 1)

\section{Conclusion}

This paper presents a work carried out using the low-level features of an image like HSV color histogram, color moments and Hu-invariant moments. Different experiments were carried out on the proposed CBIR system using the above features and combinations of the same on Wang's dataset of 1000 images of 10 classes. The performance of the system was observed using the average precision, recall, accuracy and F-score. The performance was compared with the existing works and found that the proposed system has performed better. In future work, there is a scope for improvement in proposed work by considering various other combinations of image features.

\section{REFERENCES}

[1]. Yogita Mistry D.T.Ingole, M.D.Ingole, Content based image retrieval using hybrid features and various distance metric. Journal of Electrical Systems and Information Technology, https://doi.org/10.1016/j.jesit.2016.12.009, Volume 5, Issue 3, December 2018, Pages 874-888.

[2]. Zhijie Zhao et al., Content Based Image Retrieval Scheme using Color, Texture and Shape Features. International Journal of Signal Processing, Image Processing and Pattern Recognition, http://dx.doi.org/10.14257/ijsip.2016.9.1.19, Vol.9, No.1 , 2016, pp.203-212.

[3]. Nishad P M et al., Various Colour Spaces and Colour Space Conversion Algorithms. Journal of Global Research in Computer Science, 4 (1), January 2013, 44-48.

[4]. Mr. Milind V. Lande, Prof .Praveen bhanodiya, Mr. Pritesh Jain, Efficient Content Based Image Retrieval Using Color and Texture. International Journal of Scientific \& Engineering Research, Volume 4, Issue 6, June-2013. 
[5]. G.-H. Liu et al., Content-based image retrieval using computational visual attention model, Elsevier, Pattern Recognition, 48, http://dx.doi.org/10.1016/j.patcog.2015.02.005, 2015, 2554-2566.

[6]. Purohit Shrinivasacharya, Dr. M. V Sudhamani, Content Based Image Retrieval System using Texture and Modified Block Truncation Coding. IEEE International Conference on Advanced Computing and Communication Systems (ICACCS -2013), Dec. 19 - 21, 2013, Coimbatore, INDIA.

[7]. A. Amanatiadis, V.G. Kaburlasos, A. Gasteratos, and S.E. Papadakis, Evaluation of Shape Descriptors for Shape-Based Image Retrieval, IET Image Processing, 2011, 5, (5), p. 493-499, DOI: 10.1049/ietipr.2009.0246.

[8]. Guang-Hai Liu, Jing-Yu Yang, Content-based image retrieval using color difference histogram, Elsevier, Pattern Recognition, 46, 2013, 188-198, http://dx.doi.org/10.1016/j.patcog.2012.06.001.

[9]. Ahmed J. Afifi andWesam M. Ashour, Image Retrieval Based on Content Using Color Feature. International Scholarly Research Network - ISRN Computer Graphics, Volume 2012, Article ID 248285, 11 pages, doi:10.5402/2012/248285.

[10]. L.K. Pavithra, T. Sree Sharmila, An efficient framework for image retrieval using color, texture and edge features. Elsevier, Computers and Electrical Engineering 70, 2018, 580-593.

[11]. Swapnalini Pattanaik, Prof.D.G.Bhalke, Beginners to Content Based Image Retrieval, International Journal of Scientific Research Engineering \& Technology (IJSRET), Volume 1 Issue 2, May 2012, pp 040-044.

[12]. Kemal Erdogan and Nihat Yilmaz, Shifting Colors to Overcome not Realizing Objects Problem due to Color Vision Deficiency. Proc. of the Second Intl. Conf. on Advances in Computing, Electronics and Electrical Technology - CEET 2014, ISBN: 978-1-63248-034-7 doi: 10.15224/ 978-1-63248-034-7-27, pp 011 - 014.

[13]. https://shodhganga.inflibnet.ac.in/bitstream/10603/18613/12/12_chapert-3.pdf

[14]. Jinsong Leng, Zhihu Huang, Analysis of Hu's moment invariants on image scaling and rotation. IEEE Proceedings of 2010 2nd International Conference on Computer Engineering and Technology (ICCET) Chengdu, China, http://ro.ecu.edu.au/ecuworks/6350, 2010 pp. 476-480.

[15]. Walia, E., Vesal, S. \& Pal, An Effective and Fast Hybrid Framework for Color Image Retrieval. A. Sens Imaging (2014) 15: 93. https://doi.org/10.1007/s11220-014-0093-9.

[16]. Chuen-Horng Lin, Chen Rong-Tai, and Chan Yung-Kuan, A smart content-based image retrieval system based on color and texture feature, Image and Vision Computing, Vol. 27(6), 2009,pp. 658-665.

[17]. M.M. Fraz, P. Remagnino, A. Hoppe, S.A. Barman, "Retinal image analysis aimed at extraction of vascular structure using linear discriminant classifier", Proceedings of the IEEE International Conference on Computer Medical Applications ICCMA' 2013, Jan, 2013, Sousse , Tunisia.

[18]. M.M. Fraz , A.R. Rudnicka, C.G. Owen, D.P. Strachan, S.A. Barman, "Automated Arteriole and Venule Recognition in Retinal Images using Ensemble Classification", Proceedings of the 9th International Conference on Computer Vision Theory and Applications (VISAAP), Jan, 2014, Lisbon, Portugal. 